\title{
A Review on Enhancement of Face Recognition Technology in Biometrics
}

\author{
Authors \\ Seema Mehra ${ }^{1}$, Saurabh Charaya ${ }^{2}$ \\ ${ }^{1}$ PG Student Department of Computer Science \& Engineering OM Institute of Engineering \& Technology, \\ Hisar, Haryana, India \\ ${ }^{2}$ Head of Department of Computer Science \& Engineering OM Institute of Engineering \& Technology, \\ Hisar, Haryana, India \\ Email-seemamehra05@gmail.com
}

\begin{abstract}
:
Idea for research came up while studying soft computing. Mainly research is based upon MATLAB including image processing. We have read many algorithms which are based on image processing like Adam's algorithm \& fisher's algorithm for image processing etc. images based on these algorithms as mentioned above had low quality \& contrary to this set of facts we try to improve quality of images. The objective of research is to implement Face recognition within MATLAB using edge detection mechanism like Canny. Face reorganization system is a biometric system \& this s working depends on working of biometric devices. In this research we have studied \& evaluate different edge detection techniques. We have seen that canny edge detector gives better result as compared to others with some positive points.
\end{abstract}

\section{INTRODUCTION}

\section{Face Recognition System}

A face recognition system is a group of programs for automatically recognize a person from a digital image or a video frame from a source. Ways to do this is by comparing selected characteristics from image \& face database. It is generally used within security systems \& could be compared to other identifications such as fingerprint or eye iris recognition systems. Some facial recognition applications identify facial features by extracting landmarks, or features, from an image of subject's face. For example, an algorithm could analyze relative position, size, \& shape of eyes, nose, cheekbones, \& jaw. These features are then used to search for other images with matching characteristics. Other applications normalize a group of face images \& then compress face properties, only saving data within image that is useful for face recognition. A probe image is then compared with face data. One of earliest successful systems is based on template matching techniques applied to a set of salient facial characteristics, giving a compressed face representation. Recognition algorithms could be divided into two main approaches, geometric, which overviews at important characteristics, or photometric, which is a statistical approach that distills an image into properties \& compares these properties with templates to eliminate variances.

\section{LITERATURE REVIEW}

Biometrics \& Face Recognition Techniques by Renu Bhatia Department of Computer Science \& Applications Kurukshetra University, Kurukshetra, could 2013 Biometrics is a growing technology, which has been widely used within forensics, secured access \& prison security. A biometric system is fundamentally a pattern recognition system that recognizes a person by determining authentication by using his different biological 
features i.e. Fingerprint, retina - scan, iris scan, hand geometry, \& face recognition are leading physiological biometrics \& behavioral characteristic are Voice recognition, keystroke-scan, \& signaturescan. In this paper different biometrics techniques such as Iris scan, retina scan \& face recognition technique $\mathrm{s}$ are discussed ${ }^{[2]}$ Image--based Face Detection \& Recognition: "State of Art" by Faizan Ahmad, Aaima Najam \& Zeeshan Ahmed Department of Computer Science \& Engineering, Beijing University of Aeronautics \& Astronautics Beijing, 100000, China, 2012 Face recognition from image or video is a popular topic within biometrics research. Several public places usually have surveillance cameras for video capture $\&$ these cameras have their significant value for security purpose. It is widely acknowledged that face recognition have played an important role within surveillance system as this doesn't need object's cooperation. Actual advantages of face based identification over other biometrics are uniqueness $\&$ acceptance. As human face is a dynamic object having high degree of variability within its appearance, that makes face detection a difficult problem within computer vision. In this field, accuracy \& speed of identification is a main issue. Goal of this paper is to evaluate various face detection \& recognition methods, provide complete solution for image based face detection \& recognition with higher accuracy, better response rate as an initial step for video surveillance. Solution is proposed based on performed tests on various face rich databases within terms of subjects, pose, emotions, race \& light. ${ }^{[3]}$ A Review Paper on Face Recognition Techniques Sujata G. Bhele1 \& V. H. Mankar, 2012 Sujata G. Bhele1 \& V. H. Mankar (2012) introduced A Review Paper on Face Recognition Techniques. Face recognition has been a fast growing, challenging \& interesting area within real time applications. A large number of face recognition algorithms have been developed within last decades. In this paper an attempt is made to review a wide range of methods used for face recognition comprehensively. This include PCA, LDA, ICA, SVM, Gabor wavelet soft computing tool like ANN for recognition \& various hybrid combination of this techniques.

${ }^{[4]}$ A brief experience on journey through hardware developments for image(picture)processing \& it's applications on Cryptography by Sangeet Saha, Chandrajit Pal, Rourab Paul, 2012 The importance of embedded applications on image(picture) \& video processing, communication \& cryptography domain has been taking a larger space within current research era. Improvement of pictorial information for betterment of human perception like deblurring, de-noising within several fields such as satellite imaging, medical imaging etc are renewed research thrust. Specifically we would like to elaborate our experience on significance of computer vision as one of domains where hardware implemented algorithms perform far better than those implemented through software.

\section{PROPOSED WORK}

The objective of research is to implement Face recognition within MATLAB using edge detection mechanism like Canny. Face reorganization system is a biometric system \& this s working depends on working of biometric devices. During enrolment phase, data is immediately converted into a template containing a unique binary code which represents characteristics or measurements of biometric feature. This binary code is normally encrypted \& kept within a separate storage space, for instance, within memory of biometric device. In this way biometric data is segregated from other personal information conventionally contained within a back-end database \& kept for administrative purposes. SOFTWARE REQUIREMENTS Windows 7/8 MATLAB Image processing software like Picture package manager or Photoshop. In order to implement proposed work we need work on images with Matlab. Image processing is a form of 
signal processing for which image works as input, such as a photograph or video frame; output of image processing could be either an a set of characteristics or parameters related to image. Most image-processing techniques involve treating image as a two-dimensional signal \& applying standard signal-processing techniques to it. Image processing usually refers to digital image processing, but optical \& analog image processing also are possible.

\section{TOOLS AND TECHNOLOGY USED}

Edge detection is name for a set of mathematical methods which aim at identifying points within a digital image at which image brightness changes sharply or, more formally, has discontinuities. Points at which image brightness changes sharply are typically organized into a set of curved line segments termed edges. Same problem of finding discontinuities within 1D signals is known as step detection \& problem of finding signal discontinuities over time is known as change detection. Edge detection is a fundamental tool within image processing, machine vision \& computer vision, particularly within areas of feature detection \& feature extraction.

\section{SCOPE OF RESEARCH}

The purpose of detecting sharp changes within image of face brightness is to capture important events \& changes within properties of world. It could be shown that under rather general assumptions for an image formation model, discontinuities within image of face brightness are likely to correspond .To discontinuities within Depth discontinuities within surface orientation, changes within material properties \& variations within scene illumination. In ideal case, result of applying an edge detector to an image of face could lead to a set of connected curves that indicate boundaries of objects, boundaries of surface markings as well as curves that correspond to discontinuities within surface orientation. Thus, applying an edge detection algorithm to an image of face could significantly reduce amount of data to be processed \& could therefore filter out information that could be regarded as less relevant, while preserving important structural properties of an face image. If edge detection step is successful, subsequent task of interpreting information contents within original face image may therefore be substantially simplified. However, this is not always possible to obtain such ideal edges from real life face images of moderate complexity. Edges extracted from non-trivial images of faces are often hampered by fragmentation, meaning that edge curves are not connected, missing edge segments as well as false edges not corresponding to interesting phenomena within image thus complicating subsequent task of interpreting image data. Edge detection is one of fundamental steps within image processing, image analysis, Image pattern recognition, \& computer vision techniques. There are many methods for edge detection, but most of them could be grouped into two categories, searchbased \& zero-crossing based. Search-based methods detect edges by first computing a measure of edge strength, usually a first-order derivative expression such as gradient magnitude, \& then searching for local directional maxima of gradient magnitude using a computed estimate of local orientation of edge, usually gradient direction. Zero-crossing based methods search for zero crossings within a second-order derivative expression computed from image within order to find edges, usually zerocrossings of Laplacian or zero-crossings of a nonlinear differential expression. As a pre-processing step to edge detection, a smoothing stage, typically Gaussian smoothing, is almost always applied (see also noise reduction). The edge detection methods that have been published mainly differ within types of smoothing filters that are applied \& way measures of edge strength are computed. As many edge detection methods rely on computation of image gradients, they also differ within types of 
filters used for computing gradient estimates within $\mathrm{x}-\&$ y-directions. A survey of a number of different edge detection methods could be found within (Ziou $\&$ Tabbone 1998); see also encyclopedia articles on edge detection within Encyclopedia of Mathematic \& Encyclopedia of Computer Science \& Engineering.

\section{CONCLUSIONS}

On analyzing all these edge detection techniques, this is found that canny gives optimum edge detection .Following are some points throwing light on advantages of canny edge detector as compared to other detectors discussed within this paper: Less Sensitive to noise: As compared to classical operators like Prewitt, Robert \& Sobel. Canny edge detector is less sensitive to noise. Its uses Gaussian filter which removes noise at a great extent as compared to above filters. LoG operator is also highly sensitive to noise as differentiate twice within comparison to canny operator. Remove streaking problem: classical operators' like Robert uses single thresholding technique but this results into streaking. Streaking means, if edge gradient just above \& just below set threshold limit this removes useful part of connected edge, \& leave disconnected final edge. To overcome from this drawback canny detector uses 'hysteresis' technique which uses two threshold values $t_{\text {low }} \& t_{\text {high }}$ as discussed above within canny algorithm. Adaptive within nature: Classical operator have fixed kernels so cannot be adapted to a given image. While performance of canny algorithm depends on variable or adjustable parameters like which is standard deviation of Gaussian filter \& threshold values $t_{\text {low } \&} t_{\text {high. }}$. Smaller value of $E$ results smaller Gaussian filter within turns results within finer edges. So user could changes these parameters \& could improve result of canny algorithm. Good localization: LoG operators cannot find edge orientation while canny operator provides edge gradient orientation which results into good localization.

\section{FUTURE SCOPE}

In this research we have studied \& evaluate different edge detection techniques. We have seen that canny edge detector gives better result as compared to others with some positive points. It is less sensitive to noise, adaptive within nature, resolved problem of streaking, provides good localization \& detects sharper edges as compared to others. It is consider as optimal edge detection technique hence lot of work \& improvement on this algorithm has been done \& further improvements are possible within future as an improved canny algorithm could detect edges within color image(picture)without converting within gray image, improved canny algorithm for automatic extraction of moving object within image guidance .We have used Canny Algorithm to find edges of pictures of faces \& compare them to recognition faces on bases of edges as police recognizes criminal on basis of outlines of face. While within other face recognition techniques some time picture of same person is not recognized due to change within expression, color contrast or brightness of image of face. This research is useful to compare faces on basis of edges. It finds practical application within Runway Detection \& Tracking for Unmanned Aerial Vehicle, within brain MRI image, cable insulation layer measurement, Real-time facial expression recognition, edge detection of river regime, Automatic Multiple Faces Tracking \& Detection. Canny edge detection technique is used within license plate reorganization system which is an important part of intelligent traffic system (ITS), finds practical application within traffic management, public safety \& military department. It also finds application within medical field as within ultrasound, $\mathrm{x}$-rays etc. 


\section{REFERENCES}

1. T. Ahonen \& M. Pietikäinen. Image description using joint distribution of filter bank responses.Pattern Recognition Letters, 30(4):368-376, 2009.

2. T. Ahonen, A. Hadid, \& M. Pietikainen. Face Recognition with Local Binary Patterns. Lecture Notes within Computer Science, pages 469-481, 2004

3. V. Blanz \& T. Vetter. Face recognition based on fitting a 3D morphable model. IEEE Transactions on pattern analysis \& machine intelligence, 25(9):1063-1074, 2003.

4. C. Chang \& C. Lin. LIBSVM: a library for support vector machines, 2001. Software available at http://www. csie. ntu.edu. tw/cjlin/libsvm, 2001.

5. P. Phillips, H. Moon, S. Rizvi, \& P. Rauss. FERET evaluation mthodology for facerecognition algorithms. IEEE Transactions on pattern analysis \& machine intelligence, 22(10):1090-1104, 2000.

6. Azeema Sultana, Dr. M. Meenakshi, "Design \& Development of FPGA based Adaptive Thresholder for image(picture)Processing Applications", on line access

7. Gerhard X. Ritter; Joseph N. Wilson, “ Handbook of Computer Vision Algorithms within image(picture)Algebra" CRC Press, CRC Press LLC ISBN:0849326362 Pub Date: 05/01/96

8. A. Marion An Introduction to image (picture)Processing, Chapman \& Hall, 1991

9. N. Nacereddine, Ls. Hamami, M. Tridi, \& N. Oucief , "Non-Parametric HistogramBased Thresholding Methods for Weld Defect Detection within Radiography “ ,online access.

10. Otsu,N., "A Threshold Selection Method from Gray-Level istograms,"IEEE
Transactions on Systems, Man, \& Cybernetics, Vol. 9, No. 1, 1979, pp. 62-66.

11. Elham Ashari , Richard Hornsey, " FPGA Implementation of Real-Time Adaptive image(picture)Thresholding", online access .

12. Kimmel, Ron \& Guillermo Sapiro. "The Mathematics of Face Recognition." SIAM. April 30, 2003. http://www.siam.org/news/news.php?id=309

13. Moyer, Paula. "New Technology Targets Skin as Valid Biometric Identification, Security." Dermatology Times. June 1, 2004. http://www.dermatologytimes.com/

14. The University of York, Department of Computer Science. "Biometrics: Face Recognition." http://wwwusers.cs.york.ac.uk/ nep/tomh/Biometrics.ht $\mathrm{ml}$

15. Woodward, John D, et al. "Biometrics: A Look at Facial Recognition. " RAND Public Safety \& Justice. 2003. documented_briefings/ DB396/DB396.pdf

16. Albiol,A., Albiol,A., $\quad$ Oliver,J., Mossi,J.M.(2012). Who is who at different cameras: people re- identification using depth cameras. Computer Vision, IET. Vol 6(5), 378-387.

17. "These Goofy-Looking Glasses Could Make You Invisible to Facial Recognition Technology". Slate. 18 January 2013. Retrieved 22 January 2013.

18. Meek, James (2002-06-13). "Robo cop". London: UK Guardian newspaper. "Birmingham City Centre CCTV Installs Visionics' FaceIt". Business Wire. 2008-0602 .

19. Willing, Richard (2003-09-02). "Airport anti-terror systems flub tests; Facerecognition technology fails to flag 'suspects'" (Abstract). USA Today. Retrieved 2007-09-17. 